\title{
Feed the Alien! The Effects of a Nutrition Instruction Game on Children's Nutritional Knowledge and Food Intake
}

\author{
Roel C.J. Hermans, $\mathrm{PhD},{ }^{1,2, *}$ Nina van den Broek, MSc, ${ }^{1, \star}$ Chantal Nederkoorn, $\mathrm{PhD},{ }^{3}$ \\ Roy Otten, $\mathrm{PhD}^{1,4,5}$ Emilie L.M. Ruiter, $\mathrm{PhD}$, and Mina C. Johnson-Glenberg, $\mathrm{PhD}^{5,7}$
}

\begin{abstract}
Objective: Serious games are a promising venue to increase children's nutritional knowledge in an entertaining format. The aim of this study was to test the short-term effectiveness of the Alien Health Game, a videogame designed to teach elementary school children about nutrition and healthy food choices.

Materials and Methods: To examine the effects of the Alien Health Game, an experimental design with a single between-subjects factor (experimental condition vs. active control condition) was used. A total of 108 Dutch children (10-13 years; 58 boys) were randomly assigned to either play Alien Health using the Kinect sensor for two consecutive days, for 1 hour of gameplay (experimental condition; $n=50$ ), or a web-based nutrition game for the same period (active control condition; $n=58$ ). Participants' nutritional knowledge was assessed at pretest, immediate, and at 2-week follow-up. Food intake was assessed at immediate and 2-week follow-up.

Results: Participants who played Alien Health had better knowledge of the five most important macronutrients of foods at immediate posttest, but not at follow-up. Participants were better able to distinguish the healthier food item out of two options over time, but this effect did not differ for those in the experimental versus the active control condition. No differences in food intake, neither in the consumption of nutrient-dense or energydense foods, were found between children playing both games.

Conclusion: A brief game-based intervention like the Alien Health Game has the potential to improve children's nutritional knowledge in the short term, but may not be strong enough to increase nutritional knowledge and actual eating behavior in the long term. Further investigation is warranted before this game is applied in future nutrition education programs.
\end{abstract}

Keywords: Nutrition education, Children, Serious games, Games for health

Introduction

D ESPITE CLEAR EVIDENCE that healthy eating behavior is critical for adequate development of children ${ }^{1}$ and protection against childhood overweight and obesity, ${ }^{2,3}$ many children fail to meet the recommendations for healthy nutrition. The promotion of healthy eating habits in children is therefore of utmost importance.

A potentially viable strategy to teach children about nutrition and provide them with skills to make healthy food choices is nutrition education. Nutrition education can be defined as "any combination of educational strategies designed to facilitate voluntary adoption of food choices and other food and nutrition-related behaviors conducive to health and wellbeing." ${ }^{4, ~ p . ~}{ }^{176}$ In a constant search for strategies to optimize the reach of education to children, serious games have been proposed as an effective channel for delivering health behavior change experiences and messaging in an engaging and entertaining format. 5

Although there is a growing body of literature on the potential of serious games to influence children's dietary intake or physical activity, ${ }^{5-8}$ the evidence on the effects of game-based

\footnotetext{
${ }^{1}$ Department of Developmental Psychopathology, Behavioural Science Institute, Radboud University, Nijmegen, The Netherlands.

${ }^{2}$ Department of Health Promotion, NUTRIM School for Nutrition and Translational Research in Metabolism, Maastricht University Medical Centre, Maastricht, The Netherlands.

${ }^{3}$ Department of Clinical Psychological Science, Maastricht University, Maastricht, The Netherlands.

${ }^{4}$ Research \& Development Pluryn, Nijmegen, The Netherlands.

${ }^{5}$ Department of Psychology, ASU REACH Institute, Arizona State University, Tempe, Arizona.

${ }^{6}$ Academic Collaborative Centre AMPHI, Primary and Community Care, Radboud University Medical Centre, Nijmegen, The Netherlands.

${ }^{7}$ Embodied Games, LLC, Tempe, Arizona.

*Shared first authorship.
} 
nutrition education is limited. ${ }^{9}$ Only a few studies have specifically examined whether playing a specific nutrition education game increased children's nutritional knowledge. Turnin et al., ${ }^{10}$ for instance, demonstrated that children who played several different nutritional teaching games during the nutritional teaching period increased their nutritional knowledge scores compared to those who received conventional nutritional teaching only. This pattern of results was also demonstrated by Yien, Hung, Hwang, and Lin, ${ }^{11}$ who found that the learning achievement of children playing videogames was better compared with children who learned with the traditional teaching approach. Kreisel ${ }^{12}$ and Baños et al. ${ }^{13}$ also showed that their video-based teaching tools were effective in increasing children's nutrition knowledge, but not more than the "traditional" education materials. Finally, Munguba, Valdes, and Da Silva ${ }^{14}$ showed that the simultaneous application of interactive games (e.g., videogames and board games) promoted the learning of nutritional concepts, whereas the research of Johnson-Glenberg and colleagues ${ }^{15,16}$ suggest acceptability and feasibility of the Alien Health Game to instruct in nutrition. These findings suggest that educational videogames have the capacity to increase children's nutritional knowledge.

To further assess the effectiveness of educational videogames to increase nutritional knowledge among children, there is need for high-quality research within this area. ${ }^{17}$ In this study, we sought to test the effects of the Alien Health Game on children's nutritional knowledge with research design elements that address limitations of our previous work. ${ }^{15,16}$ Furthermore, we wanted to gain more insight in its relative effectiveness compared to conventional approaches.

This study will extend previous work in three important ways. First, we will test the effectiveness of the Dutch version of the game. This newly developed version has more (Dutch) food choices and the short cardio exercises have been replaced by two short sections where players lunge left and right to guide a spaceship. Thus, there is more focus on nutrition and food choice and less on exercise. Second, an active control condition is included in the experimental design to adequately compare the effects of this game with a web-based nutrition game that has been available for children to play. Active control groups, rather than no-contact control groups, are essential to ensure that attention, motivation, and expectations do not account for improvements in the experimental condition. ${ }^{18}$ Because of the more immersive and engaging character of Alien Health and the specific knowledge elements (i.e., making forced choice decisions on basis of the nutritional profiles of existing and nonexisting foods) incorporated in the game, it is hypothesized that children playing Alien Health would show increased nutritional knowledge at immediate and 2-week follow-up compared to those in the active control condition. Third, as a secondary outcome measure, we examined whether children in both conditions differed in food intake directly after playing the game and at 2-week follow-up. This is a timely topic because there are questions regarding whether short bouts of gameplay can have longer-term behavioral effects. ${ }^{17}$

\section{Materials and Methods}

\section{Design and participants}

An experimental design with a single between-subjects factor (experimental condition vs. active control condition) was used.
Participants in the experimental condition were asked to play the Alien Health Game, whereas children in the active control condition played Super Shopper. The Alien Health Game is developed by Embodied Games for Learning, LLC. ${ }^{15,16}$ Please consult Table 1 for a detailed description of the game and Figures 1-4 for screenshots of the different game modes.

Super Shopper is a web-based Flash game developed by IJsfontein, which uses still images without active animations. The control game was selected on its age-appropriateness and its inclusion of nutritional information. Unlike Alien Health, however, Super Shopper was not explicitly designed to educate children in healthy food choices and the main function of the five most important macronutrients. When playing Super Shopper, children are asked to select specific groceries (e.g., fruits or vegetables), after which they receive information on the nutritional values of these products. The game was played on a 16-inch laptop. Please consult Figure 5 for a screenshot of this game.

A convenience sample of three primary schools in the southern part of the Netherlands was selected. These schools were invited to participate in this study because they took part in the universal prevention program "KERNgezond," a school-based lifestyle program aimed at children between 8 and 12 years. Teachers of grade five and six of these three schools were contacted by telephone and informed about the study. Additional information was sent by e-mail to the headmaster of their schools.

A total of 118 fifth and sixth graders were recruited with active informed consent of their caregivers. Only children who received permission from their parents and those who were without medical conditions that could affect their food intake (e.g., food allergies) were able to participate in this study. Ten children were excluded afterward: five children did not or could not complete the nutritional knowledge tests (e.g., due absence or language issues), four children were absent during one of the play sessions, and one child was not able to play the game properly due to technical issues. Therefore, the final sample consisted of 108 children (58 boys and 50 girls) aged 10 to 13 years.

\section{Procedure}

Children in both conditions played their videogame twice for $\sim 30$ minutes, on two consecutive days at their school. Using the student roster of the school classes that volunteered in our study, we randomly assigned all children, one by one, to one of both conditions, while taking care that boys and girls were equally represented in both conditions. All participants were blinded to the study hypotheses. Children played their videogame individually, with another child playing the same game in the same room. A different room was used for the experimental and active control group. By doing so, we could test four children per time slot (i.e., two children per condition). During all play sessions, two research assistants were present to give instructions or to answer questions related to the gameplay.

All participants completed the nutritional knowledge test at three time points: before the first play session (i.e., pretest), immediately after the second play session (i.e., posttest), and at 2-week follow-up. Children's height and weight were assessed before the first play session. After the second play session and at 2-week follow-up, participants 


Health topic(s)
Targeted age groups(s)
Other targeted group characteristics
Short description of game idea
Target player(s)

Guiding knowledge or behavior change theory(ies), models, or conceptual framework(s)

Intended health behavior changes

Knowledge element(s) to be learned

Behavior change procedure(s) (taken from Michie inventory)

Clinical or parental support needed?

Data shared with parent of clinician?

Type of game: (check all that apply)

Story (if any) Synopsis (including story arc)

How the story relates to the targeted behavior change

Game components

Player's game goal/objective(s)

Rules
Dietary intake; nutrition education

Elementary school children (8-12 years).

Children must be able to read.

Players are instructed about the nutritional profiles of foods as they keep an Alien pilot healthy to fly his ship.

$\square$ Individual $\square$ Dyad $\square$ Small group MMOG $\square$ Other:

Situated and embodied cognition

Increase nutritional knowledge; stimulate healthy food choices.

Players learn how to read the nutritional profiles of everyday foods and the main function of the five most important macronutrients (i.e., fat, fiber, protein, vitamins, and carbohydrates).

Praise for correct choices, feedback on performance, and prompt to rehearse and practice with food choices for the Alien.

$\square$ Yes $\square$ No

Yes $\square$ No

$\square$ Active $\square$ Action $\square$ Adventure $\square$ Role-Playing $\square$ Simulation $\square$ Strategy $\square$ Sports $\square$ Casual $\square$ Educational $\square$ Other:

A foundling alien must be kept healthy to save other dying planets. To do so, players need to make forced choice decisions on basis of the nutritional profiles of existing and nonexisting foods. Depending on the food choice, the alien either perks up or becomes more fatigued. There are six stages of feedback until the alien is fully energized ("super fit"). When the alien has enough energy, he is able to fly his bio-linked ship to complete his missions.

By figuring out the healthiest foods for the Alien, players are praised and receive constant feedback and knowledge about the nutritional profiles of common foods. The hypothesis is that this will translate into real-world better food choices.

To keep the Alien as healthy as possible to fly his spaceship through the asteroid field and save the planet.

Forced Choice Game Mode 1

- Player must choose the healthiest food between 2 presented options

- Player may not cancel or change a previous choice

- Food choices change the state of the Alien

- The Player wins when the Alien is in the "Super Fit" state

See Figure 1 for a screenshot of this mode.

Quick Sort Game Mode 2

- Player must choose the healthiest food between 2 presented options

- Player may not cancel or change a previous choice

- A countdown timer counts down to 0

- Game ends when all pairs of food are sorted or the timer hits 0

See Figure 2 for a screenshot of this mode.

Build a Meal Game Mode 3

- Player is presented with a carousel of foods organized by Main course, Side dish, drink \& dessert

- Player must choose one food from each carousel to place on the plate

- Healthier foods chosen award the player with bonus shields for the alien's ship

See Figure 3 for a screenshot of this mode.

Ship Runner Game Mode 4

- Player jumps at least $1 \mathrm{~m}$ to the left or the right to steer the ship left or right

- Hitting an asteroid reduces shields by 1

- Coins give the player points

- Seeds are needed to win the level

- Player wins when they reach the end of the level with specified number of seeds

- Player loses when all shields are gone

See Figure 4 for a screenshot of this mode. 
Table 1. (Continued)

Game mechanic(s)

Procedures to generalize or transfer what is learned in the game to outside the game

Virtual environment

Setting (describe)

Avatar

Characteristics

Abilities

Game platform(s) needed to play the game

Sensors used

Estimated play time
Food Selection Game Mode

- Binary Choices to reach a goal

Quick Sort Game Mode

- Rapid Binary Choices to assess nutrition comprehension and rapid decisionmaking

Build a Meal Game Mode

- Cumulative Choices to build a set of foods that benefit or harm the player's survival chances in the Ship Runner Game Mode

Ship Runner Game Mode

- Goal Runner on 3 rails.

- Player uses embodied gestures to steer ship

- Timing to avoid obstacles and collect coins and seeds

- Survive to reach the goal

Praise for correct choices, feedback on performance, prompt to rehearse and practice with food choices for the Alien, and ability for replay after the test phase.

The VR environment inside the space ship and outerspace, in an asteroid field.

Cute and silent, the avatar is a blue/purple alien that expresses his health and wellbeing through facial and body animations.

The alien avatar is never directly controlled by the player. It is an NPC with no specific abilities. The alien's animations serve as both positive and negative feedback for the player when choosing foods
$\square$ Smartphone $\square$ Tablet $\square$ Kinect Xbox $\square$ Wii

Handheld device $\square$ Other:

Microsoft Xbox One Kinect 2.0

1 hour, best broken into two play sessions. participated in a food-taste test. This test served as a proxy for children's real-world food intake, which is a common procedure in eating behavior research (e.g., Guerrieri et al. $\left.{ }^{19}\right)$. The study and its procedures were approved by the ethics committee of the Faculty of Social Sciences, Radboud University with registration no. 2016-2501-371. All data were collected between January and June 2016.

\section{Measures}

Height and weight. The experimenter measured individual height and body weight of all participants according to standard procedures (i.e., without shoes, but fully clothed). Height was measured to the nearest of $0.1 \mathrm{~cm}$ using a stadiometer (Seca 217 Slider; Seca GmbH \& Co., Hamburg,

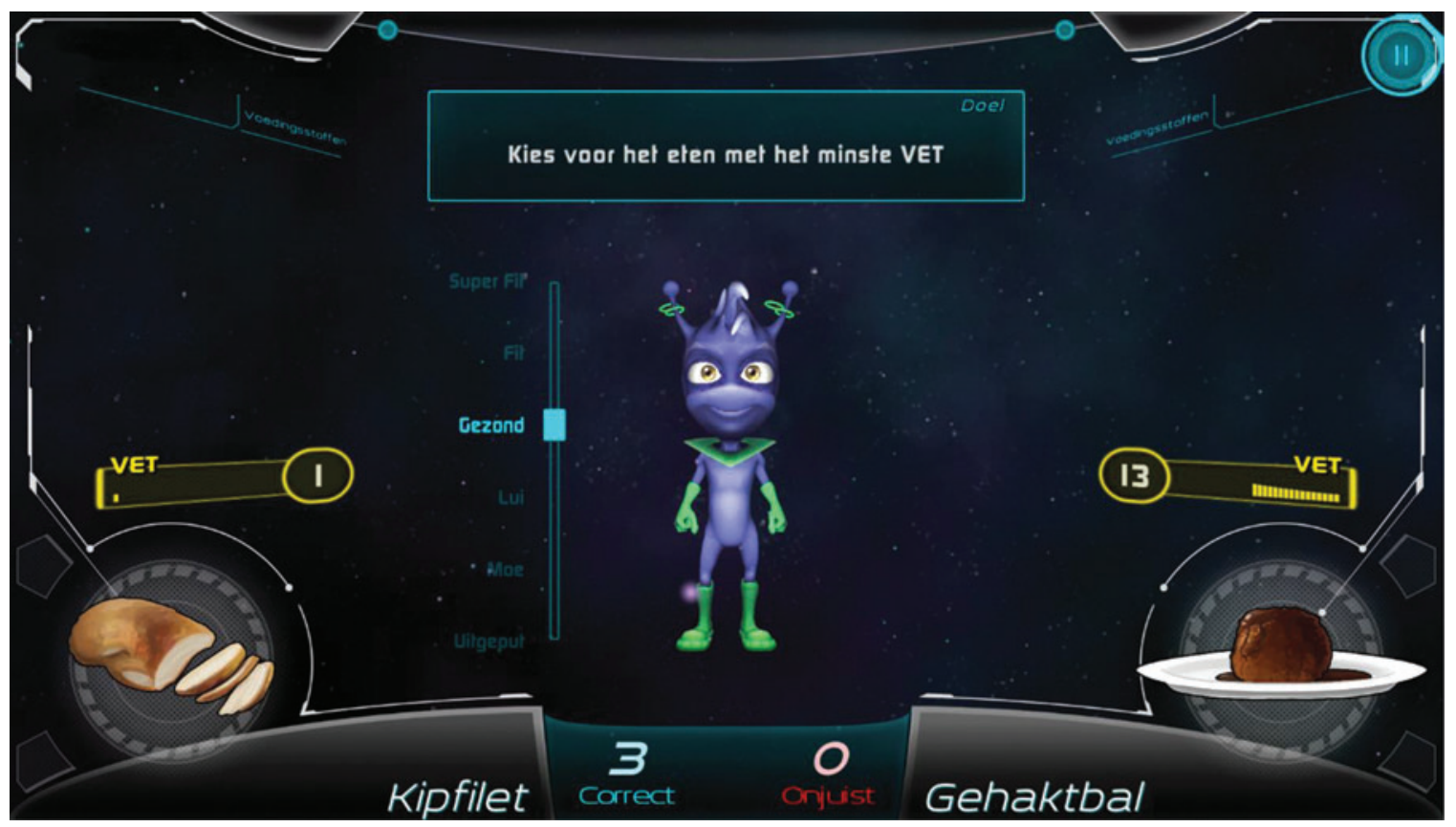

FIG. 1. Screenshot of Forced Choice Game Mode 1. Color images available at www.liebertonline.com/g4h 


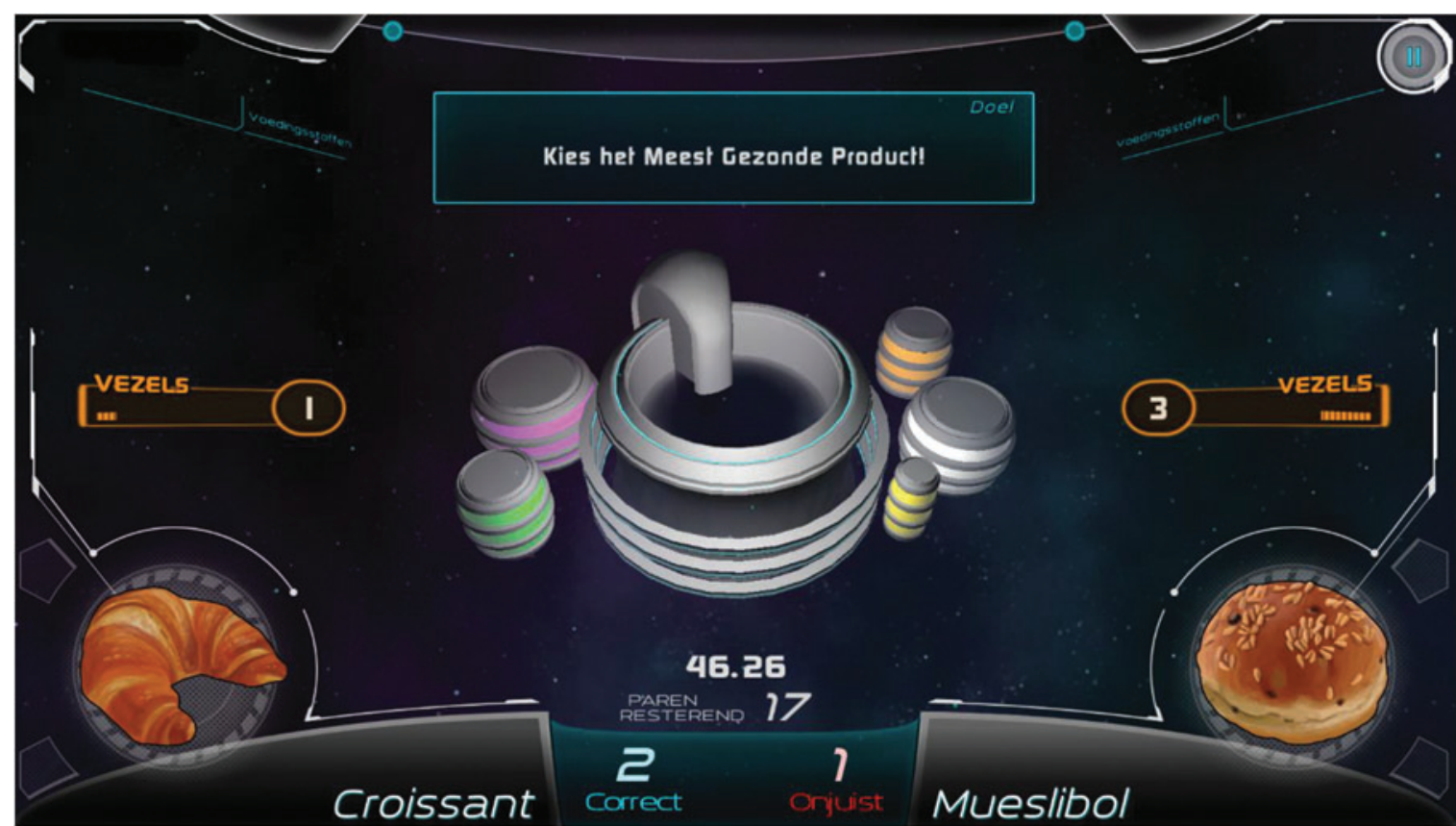

FIG. 2. Screenshot of Quick Sort Game Mode 2. Color images available at www.liebertonline.com/g4h

Germany) and weight was measured to the nearest of $0.1 \mathrm{~kg}$ using a digital scale (Seca Bella 840; Seca GmbH \& Co.). Age- and gender-standardized BMI (zBMI) scores were calculated according to Centers for Disease Control and Prevention guidelines. ${ }^{20}$

Nutritional knowledge. Children's nutritional knowledge was assessed in three ways. First, an open-ended question was used in which children were asked to name the five most important macronutrients of foods (i.e., vitamins, fiber, car- bohydrates, protein, and fat). For every correct macronutrient, children earned one point. A total score (range 0-5) was created to use as a dependent variable in the analyses. Second, a multiple-choice paradigm was used in which children were asked to identify the main function of each of the five macronutrients. They were asked to choose between one of four options (e.g., main function of fat is: "they are the building blocks of your body", "they help your body with growing and recovery", "they provide energy", and "they improve the functioning of your brains"). For every correct

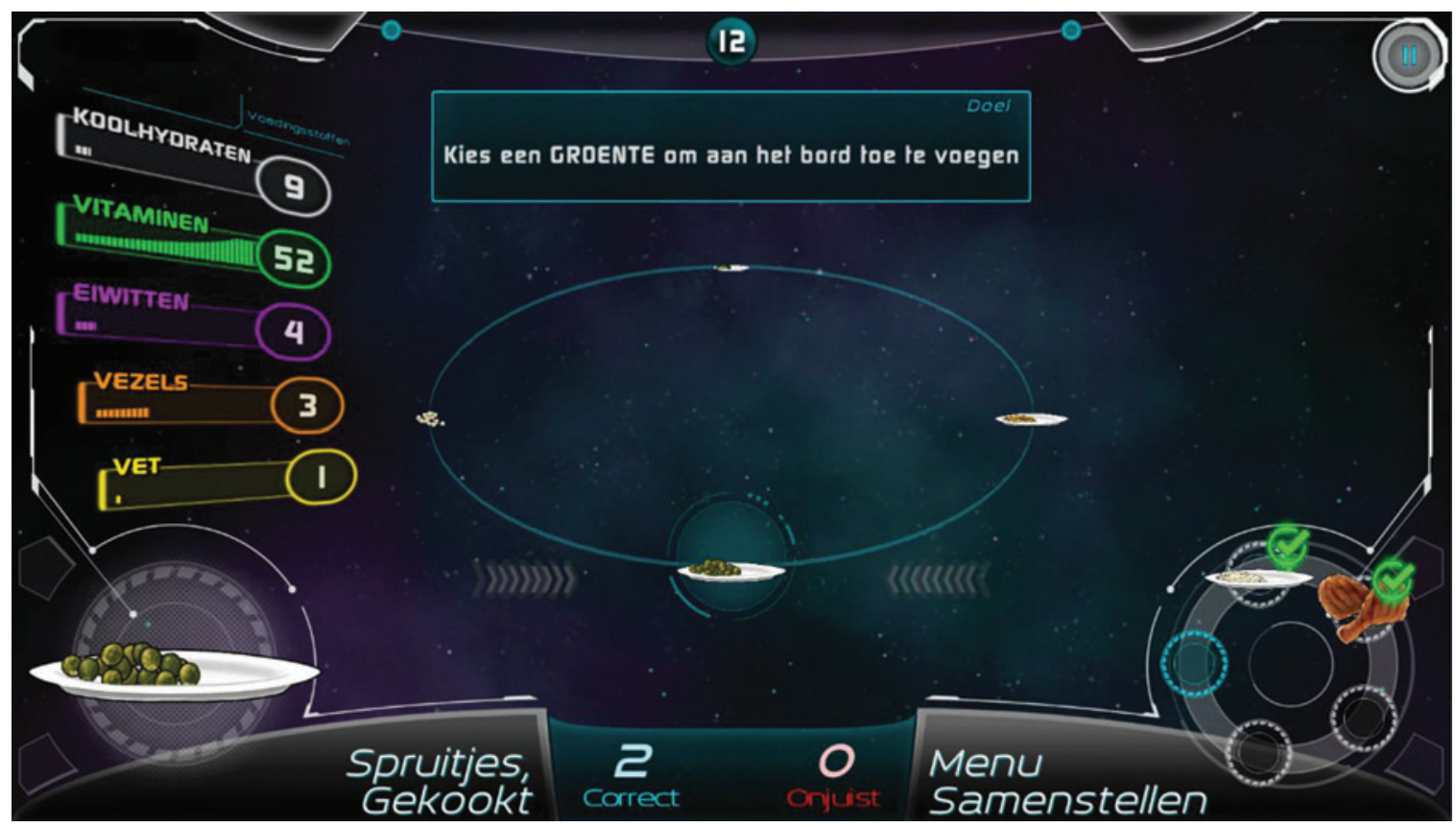

FIG. 3. Screenshot of Build a Meal Game Mode 3. Color images available at www.liebertonline.com/g4h 


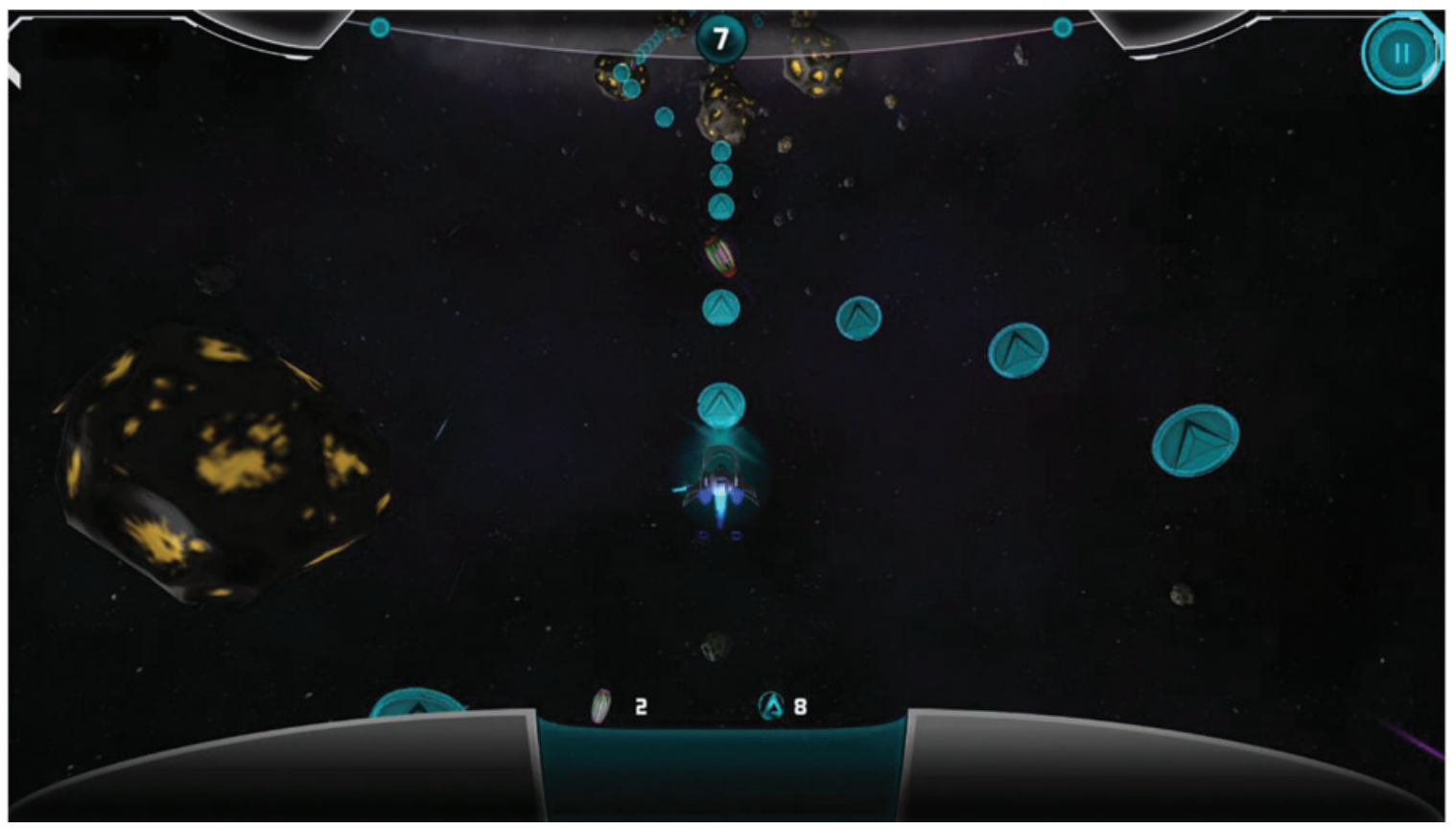

FIG. 4. Screenshot of Ship Runner Game Mode 4. Color images available at www.liebertonline.com/g4h

answer, children earned one point. Again, the total score (range 0-5) was used in the analyses. Finally, children had to choose the "healthier food item" out of two options (e.g., white bread vs. wheat bread; grape juice vs. grapes; corn flakes vs. oat meal; and iced tea vs. tea without sugar). This measure included 20 forced-choice questions. For every correct answer, children earned one point. Children's total score (0-20) was used as a dependent variable.

Food intake. Under the pretext of a taste test, all children were given the opportunity to eat from an assortment of foods. The foods offered were either high energy dense or nutrient

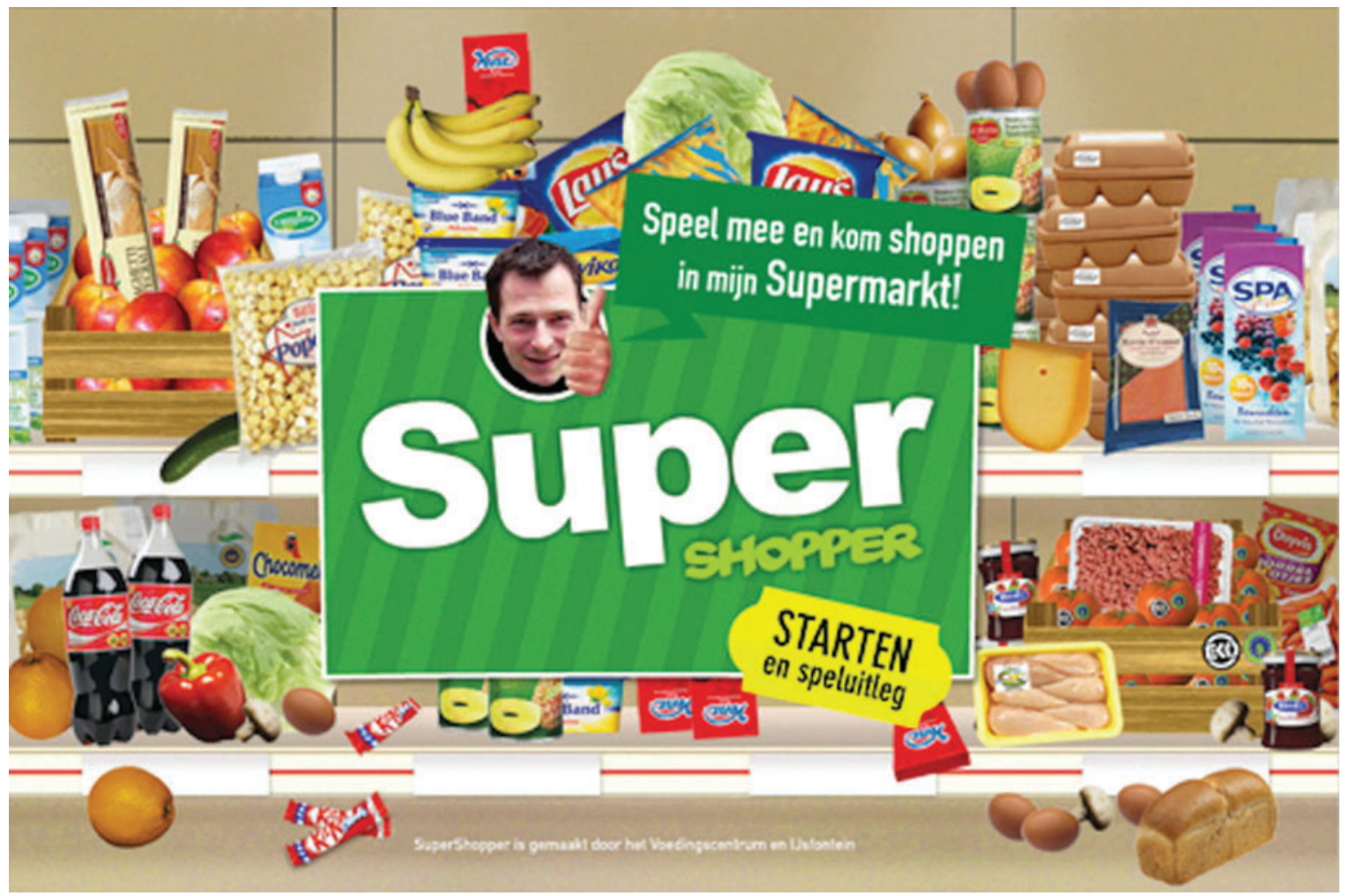

FIG. 5. Screenshot of the Control Game "Super Shopper." Color images available at www.liebertonline.com/g4h 
dense. The high energy-dense foods were salted potato crisps $(5.47 \mathrm{kcal}$ per gram) and chocolate candies $(4.86 \mathrm{kcal}$ per gram). The nutrient-dense foods were green grapes $(1 \mathrm{kcal}$ per gram) and cucumber slices ( $0 \mathrm{kcal}$ per gram). To measure children's food intake, the food was weighed before and after each test. The total amounts of food consumed in grams from the high energy- and nutrient-dense foods were used as two separate dependent variables in the secondary analyses.

\section{Strategy of analyses}

First, randomization checks were performed to assess whether any differences exist between conditions for potential confounders (i.e., age, gender, and zBMI) and the main dependent variables. Since independent samples' $t$-tests are considered not appropriate to perform randomization checks, ${ }^{21}$ we used Cohen's $d$ to assess whether differences between conditions can be interpreted as trivial (i.e., Cohen's $d \approx 0.20$ ). Second, to answer the main research questions, five repeatedmeasures analyses of variance (RM ANOVAs) were conducted to (1) assess the effect of condition on nutritional knowledge (i.e., macronutrient knowledge, macronutrient function, and healthier food item) and (2) assess the effect of condition on food intake (i.e., high energy-dense vs. nutrient-dense food intake).

Several statistical assumptions were checked. These included multivariate normality (checked using inspection of distributions of the dependent variables), the absence of outliers (i.e., standardized values larger or smaller than 3 and -3 , respectively), sphericity (checked using Mauchly's test of sphericity), homogeneity of variances (checked using Levene's test for equality of error variances), and homogeneity of covariances (checked using Box's $M$ test for equality of covariances). Significant outliers were winsorized to the next value in the normal range.* Effect sizes of the reported effects were expressed as partial eta squares (i.e., $\eta^{2}$ partial) and were interpreted as small, medium, and large based on the values $0.02,0.13$, and 0.26 , respectively. ${ }^{22}$ Alpha was set at $P<0.05$. All reported $P$ values were corrected for multiple testing using a Bonferroni correction.

\section{Results}

\section{Randomization checks}

Table 2 presents mean values for participant characteristics, separately for the experimental condition and the control condition.

No differences were found in participants' age, gender, or zBMI between the two conditions. Moreover, no differences were observed in participants' nutritional knowledge, number of days between pretest and posttest, and timing of taste test, implying successful randomization.

\section{Main effects: the effect of condition on nutritional knowledge}

Macronutrient knowledge. Findings indicated a significant effect of time, $F(2,204)=91.47, \quad P<0.001$, and $\eta_{\text {par- }}^{2}$

*With regard to children's nutrient-dense food intake, two outliers were detected and winsorized. With regard to children's energydense food intake, three outliers were detected and winsorized. Results did not change when outliers were winsorized or not.
Table 2. Descriptives of Experimental Condition and Control Condition $(N=108)$

\begin{tabular}{lcc}
\hline Variables & $\begin{array}{c}\text { Experimental } \\
\text { condition } \\
(\mathrm{n}=58)\end{array}$ & $\begin{array}{c}\text { Control } \\
\text { condition } \\
(\mathrm{n}=50)\end{array}$ \\
\hline Age & 11.48 & 11.30 \\
Gender ( $\mathrm{n}$ boys) & 31 & 27 \\
zBMI & -0.01 & -0.15 \\
$\begin{array}{l}\text { Macronutrient knowledge } \\
\text { pretest (score 0-5) }\end{array}$ & 1.11 & 1.33 \\
$\begin{array}{l}\text { Macronutrient function } \\
\quad \text { pretest (score 0-5) }\end{array}$ & 2.44 & 2.68 \\
$\begin{array}{l}\text { Healthy food choice } \\
\text { pretest (score 0-20) }\end{array}$ & 14.55 & 15.08 \\
$\begin{array}{l}\text { Number of days between } \\
\text { pretest and posttest }\end{array}$ & 1.19 & 1.22 \\
$\begin{array}{l}\text { Time of taste test at posttest } \\
\text { Time of taste test at follow-up }\end{array}$ & $10.40 \mathrm{am}$ & $10.51 \mathrm{am}$ \\
\hline
\end{tabular}

tial $=0.473$, but not of condition, $F(1,102)=5.70, P=0.323$, and $\eta_{\text {partial }}^{2}=0.053$.

Furthermore, a significant time by condition interaction, $F(2$, $204)=11.94, P<0.001$, and $\eta_{\text {partial }}^{2}=0.10,5$ was found. This interaction indicates that the change in knowledge of macronutrients (i.e., fat, fiber, protein, vitamins, and carbohydrates) differed between the conditions. Independent samples' $t$-tests showed that children who played the Alien Health Game had better knowledge at immediate posttest, $t(105)=4.45$, $P<0.001$, but not at 2-week-follow-up, $t(105)=1.30, P=0.999$. Figure 6 displays the change of nutritional knowledge over time, for both conditions separately.

Macronutrient function. No effect of time, $F(2,210)=$ $0.652, P=0.999$, and $\eta^{2}$ partial $=0.006$, or condition was found, $F(1,105)=0.014, P=0.999$, and $\eta_{\text {partial }}^{2}<0.001$. Furthermore, no interaction was found between time and condition, $F(2,210)=1.501, P=0.999$, and $\eta_{\text {partial }}^{2}=0.014$.

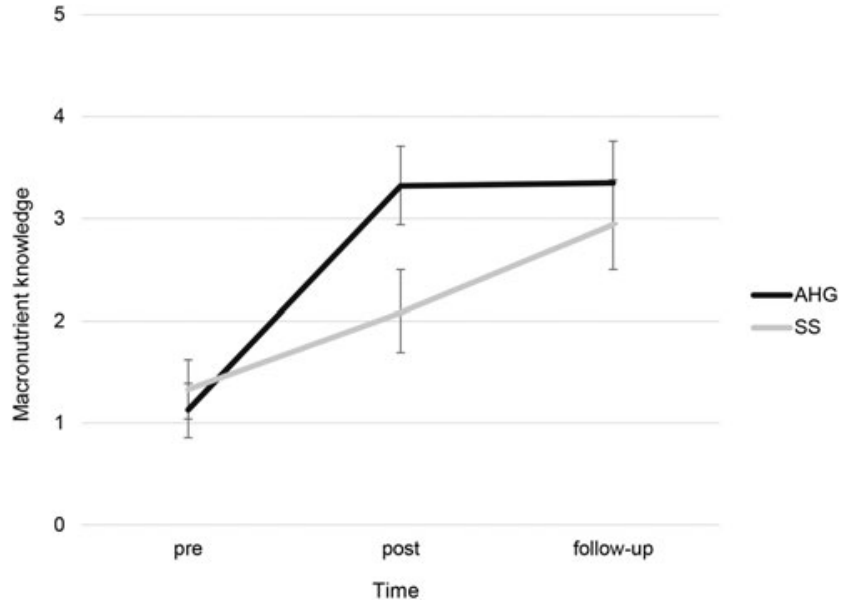

FIG. 6. Knowledge of the five macronutrients over time, separately for the experimental condition (AHG) and the control condition (SS). Error bars reflect the $95 \%$ confidence interval. AHG, Alien Health Game; SS, Super Shopper. 
Healthier food item. A significant effect of time, $F(2$, $188)=6.80, P=0.024$, and $\eta_{\text {partial }}^{2}=0.067$, but not of condition was found, $F(1,94)=0.716, P=0.999$, and $\eta_{\text {partial }}^{2}=0.008$. No interaction was found between time and condition, $F(2,188)=0.814, P=0.999$, and $\eta^{2}$ partial $=0.009$. Thus, participants were better able to select the healthier food item out of two options over time, but this effect did not differ for those in the experimental versus the control condition. Figure 7 displays the change in food choices over time, separately for both conditions.

\section{Secondary effects: the effect of condition on food intake}

Nutrient-dense food intake. No effect of time, $F(1,105)=$ $4.19, P=0.731$, and $\eta_{\text {partial }}^{2}=0.038$, or condition, $F(1,105)=$ $0.400, P=0.999$, and $\eta_{\text {partial }}^{2}=0.004$, was found. Furthermore, no interaction was found between time and condition, $F(1,105)=0.259, P=0.999$, and $\eta_{\text {partial }}^{2}=0.002$. Figure 8 displays the consumption of nutrient-dense food (in grams) over time, separately for both conditions.

Energy-dense food intake. A main effect of time was found, $F(1,105)=83.10, P<0.001$, and $\eta^{2}$ partial $=0.442$, indicating that participants in both conditions ate more energy-dense foods at 2-week follow-up than at immediate posttest. The main effect of condition was not significant, $F(1,105)=1.14, P=0.999$, and $\eta_{\text {partial }}^{2}=0.011$, neither was the time by condition interaction, $F(1,105)=0.037$, $P=0.999$, and $\eta^{2}$ partial $<0.001$. Figure 9 displays the consumption of energy-dense foods (in grams) over time, separately for both conditions.

\section{Discussion}

This study examined the short-term effectiveness of the Alien Health Game, a videogame designed to teach elementary school children about nutrition and healthy food choices. Children who played the Alien Health Game had better recall of the five most important macronutrients of

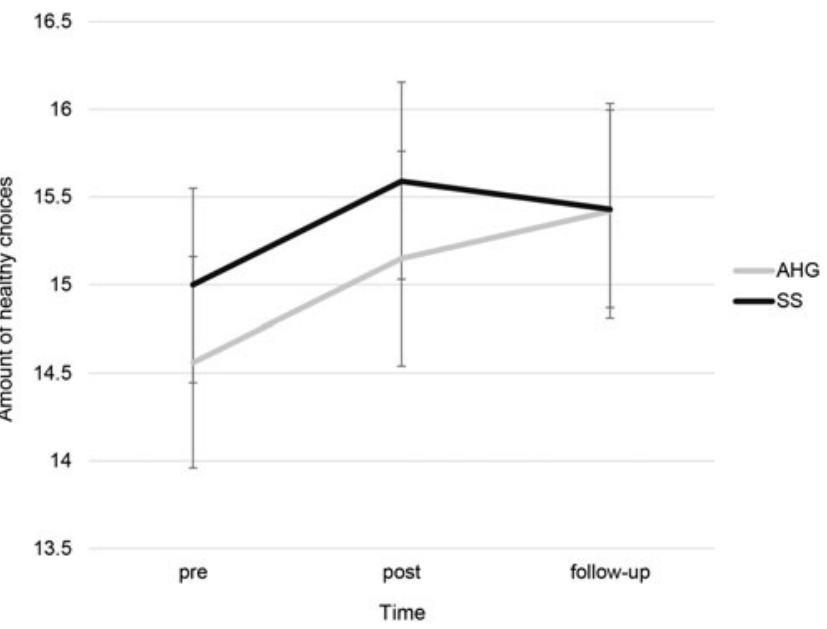

FIG. 7. Number of correct answers in the forced-choice healthiest food item task over time, separately for the experimental condition (AHG) and the control condition (SS). Error bars reflect the $95 \%$ confidence interval.

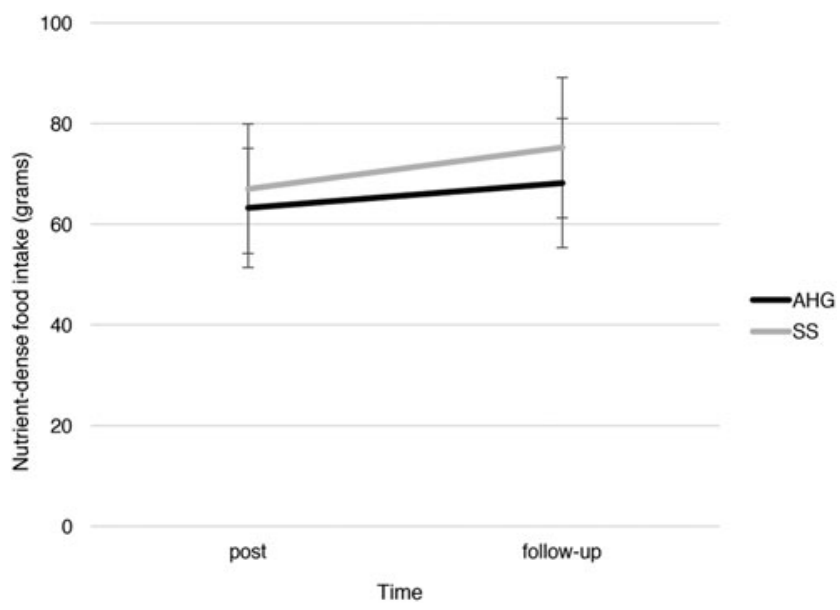

FIG. 8. Intake of nutrient-dense food (in grams) over time, separately for the experimental condition (AHG) and the control condition (SS). Error bars reflect the $95 \%$ confidence interval.

foods directly after the game compared to children in the active control group. At 2-week follow-up, however, children in both conditions performed similarly on this part of the test. Neither of the two games increased children's knowledge of the function of these macronutrients. Children were better able to select the healthier food item out of two options over time, but this effect did not differ between games. Finally, no differences in food intake, neither in the consumption of nutrient-dense or energy-dense foods, were found between children playing both games. Yet, it was found that, on average, all children ate more energy-dense food at the second session compared to the first session.

Although the results of this study demonstrate that the Alien Health Game increased certain aspects of children's nutritional knowledge in the short term, a few considerations warrant further discussion. Despite the engaging and immersive gameplay and the specific knowledge elements that were incorporated in the Alien Health Game, players did not

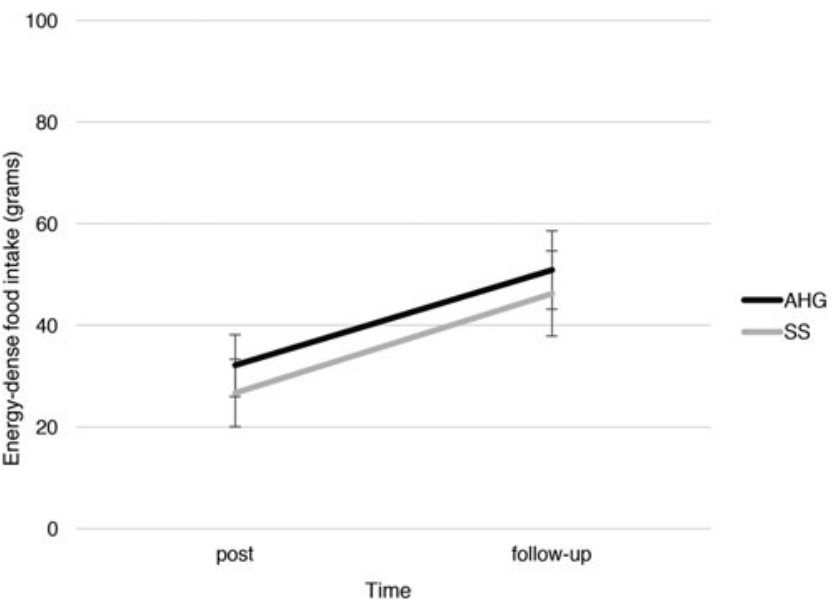

FIG. 9. Intake of energy-dense food (in grams) over time, separately for the experimental condition (AHG) and the control condition (SS). Error bars reflect the $95 \%$ confidence interval. 
outperform those playing the Super Shopper game. Although children in the experimental condition showed better immediate recall of the main function of macronutrients than those in the control condition, strong retention was not seen in the 2-week delayed test. Furthermore, no differences were found in children's knowledge of the function of the five most important macronutrients. This was unexpected given that these concepts were specifically targeted in the experimental condition, but not in the control condition. It should be noted, however, that children in the active control group were still exposed to the nutritional values of different food products in the game. Furthermore, the results of the pretest showed that children in both groups were, on average, already quite knowledgeable. Thus, there might not have been much room for growth. Finally, all children filled in the same knowledge test for three times in a short period of time (i.e., 2 weeks). The potential learning effects that might have occurred as a result of this exposure could have improved children's knowledge in both groups similarly.

There were also several issues associated with the knowledge elements and interactivity that might have attenuated potential learning effects in the experimental group compared to the control group. In the U.S. version of the game, ${ }^{16}$ the information on the macronutrient content of food was embedded within the MyPlate guidelines, the U.S. government's primary food group symbol to help consumers adopt healthy eating habits consistent with the Dietary Guidelines for Americans. Unfortunately, it was not possible to specifically include information on the Dutch equivalent of MyPlate (i.e., The Wheel of Five ${ }^{23}$ ) to the game. Without this context, Alien Health's current focus on macronutrients and its functions might have been too abstract or difficult for children to recall and induce strong learning effects. Furthermore, Alien Health was driven by participants holding their arms in front and using an "open-and-close-the fist" gesture recognized by the Kinect interface to advance each screen. Children's gestures were specifically chosen as game input because body activity can be an important catalyst for generating learning. ${ }^{24}$ Yet, game observers saw that the repeated movement of "grabbing" was tiresome for some children, making it likely that the players' fatigue may have negatively affected engagement and concentration.

In contrast to the previous version of the game, the only full body activity is lunging left and right to guide a ship at four times. It would be interesting to change the grabbing gesture, include more body movements, and to follow up for longer periods of time to see whether the new game would become (more) effective over time and how much gameplay (dosage) would be most effective in increasing nutritional knowledge. This, however, would require extensions and adaptations to the existing game (e.g., adding content and improved gameplay to stimulate interactivity and immersion).

An important secondary aim of this study was to establish whether Alien Health would positively influence children's food choice and intake. Our results, however, indicate that two short bouts of gameplay do not induce longer-term behavioral effects. In contrast, it was found that children consumed more energy-dense foods at the follow-up measurement compared to the first measurement. It is possible that children's initial experience with the first taste test has guided their intake at the second test. During the first test, they may have noticed that they were allowed to eat as much as they wanted from the energy-dense, "unhealthy," foods, without any restrictions from parents, teacher, or experimenter, which might have set a personal norm for appropriate intake during the second test. $^{25,26}$ Although our findings might suggest that children preferred the energy-dense foods over the nutrient-dense foods, we are not certain about this. Because the test foods differed in weight and energy content, we could not accurately (and directly) compare children's consumption of energydense and nutrient-dense foods during one session. To assess whether children indeed consume more energy-dense foods compared to nutrient-dense foods, future research could specifically count the number of individual foods consumed during the sessions.

An important strength of this study was the use of scientifically rigorous research and statistical methods to conduct the research and analyze data, thereby contributing to a stronger empirical base for games for health in general, and nutrition education in particular. ${ }^{17}$ Despite these strong points, a few limitations should be mentioned. First, without the inclusion of a no-contact (e.g., waitlist) control group, we cannot make the claim that the change in nutritional knowledge of both conditions is greater than what would be expected from receiving any nutritional information at all (see also Schoneveld et al. ${ }^{27}$ ). Therefore, future studies could include a waitlist control condition to effectively compare conditions. Second, given the lack of weight-related diversity in our sample, we cannot generalize our results for children with overweight or obesity. Finally, because randomization was performed on an individual level within schools, we cannot rule out the possibility that there might have been contamination between the groups. Although a different room was used for the experimental and active control group, the nutritional information learned in Alien Health may have transferred across groups and may account for the similar improvements in the control condition.

In conclusion, our findings suggest that two short bouts of Alien Health can increase some types of knowledge in the short term, but may not be strong enough to significantly increase children's nutritional knowledge and actual eating behavior in the long term. ${ }^{4,17,28}$ This should not be too surprising, because nutrition knowledge forms only one element of the complex set of skills, knowledge, and behaviors that is required to plan, manage, select, prepare, and eat food to meet needs and determine intake. ${ }^{29}$ In addition, external factors such as food parenting practices or home food availability exert a strong influence on children's food-related knowledge and behavior. ${ }^{30}$

A valuable addition to our game, therefore, would be to have a broader conceptualization of the behavioral skills that are needed to make proper food choices. Within this context, we could also focus more on theory-based determinants, such as attitudes and motivations, and include practical applications that target the behavioral skills relevant to making proper food choices. This new version, for instance, could include the shared family environment as a unique opportunity to empower and improve parents' and children's foodrelated behaviors.

In the Netherlands, nutrition education in primary schools is not mandatory and has to compete with many other subjects, as schools have a busy schedule with a major focus on core subjects. ${ }^{31}$ Videogames might overcome these barriers and they show potential to develop themselves as innovative approaches to health education and behavior change. This study demonstrates that even short bouts of gameplay can 
increase some aspects of children's nutritional knowledge. On basis of these results, however, we need to conclude that Alien Health needs further adaptations with regard to the gameplay and content before it can become a credible part of Dutch school-based nutrition education. If Alien Health is to truly impact children's nutritional knowledge and eating behavior in the long term, then it is critical that we extent gameplay and focus more on the behavioral determinants and skills that are needed to make healthy food choices.

\section{Acknowledgments}

This work was supported by a grant of the Province of Limburg, The Netherlands. The authors would like to thank Ken Koontz and James Comstock for all the effort they put into the development of the game and technical help during data collection. In addition, we would like to thank Sophie Luderer who helped us to choose the best foods to display in the game and to develop the nutritional knowledge test. Finally, we would like to thank Maurice Graef and Ellen Thijssen from Sport-Company for their help with recruiting the schools involved in this study and Joni Scholte, Anke Mertens, Eveline Dijkhuizen, and Leanne Hanegraaf for their help with collecting the data.

\section{Authors' Contributions}

Secured funding for the research: R.C.J.H., R.O., E.L.R., and C.N.; designed the experiment: R.C.J.H.; data analysis: Nv.dB; wrote the article: R.C.J.H. and Nv.dB.; provided general advice on the article: M.J.-G., R.O., and C.N.

\section{Author Disclosure Statement}

Alien Health Game has been developed by Embodied Games, LLC. Mina Johnson-Glenberg has founded this company. However, Alien Health Game is not commercialized.

It can be accessed for free under Alien Experiment at https://www.embodied-games.com/games/all/alien-health. The chance of competing interests is only present in case of commercialization of this game. Hermans, Van den Broek, Otten, Ruiter, and Nederkoorn declare that they have no competing financial interests.

\section{References}

1. Johnson R, Nicklas T. Position of the American Dietetic Association: Dietary guidance for healthy children aged 2 to 11 years. J Am Diet Assoc 2004; 104:660-677.

2. Epstein LH, Paluch RA, Beecher MD, et al. Increasing healthy eating vs. reducing high energy-dense foods to treat pediatric obesity. Obesity 2008; 16:318-326.

3. Guo X, Warden BA, Paeratakul S, Bray GA. Healthy eating index and obesity. Eur J Clin Nutr 2004; 58:15801586.

4. Contento IR. Nutrition education: Linking research, theory, and practice. Asia Pac J Clin Nutr 2008; 17:176-179.

5. Baranowski T, Buday R, Thompson DI, Baranowski J. Playing for real: Video games and stories for health-related behavior change. Am J Prev Med 2008; 34:74-82.

6. Guy S, Ratzki-Leewing A, Gwadry-Sridhar F. Moving beyond the stigma: Systematic review of video games and their potential to combat obesity. Int J Hypertens 2011; 2011:179124.
7. Lu AS, Kharrazi H, Gharghabi F, Thompson D. A systematic review of health videogames on childhood obesity prevention and intervention. Games Health J 2013; 2:131141.

8. Parisod H, Pakarinen A, Kauhanen L, et al. Promoting children's health with digital games: A review of reviews. Games Health J 2014; 3:145-156.

9. Beasley N, Sharma S, Shegog R, et al. The Quest to Lava Mountain: Using video games for dietary change in children. J Acad Nutr Diet 2012; 112:1334-1336.

10. Turnin M, Tauber M, Couvaras O, et al. Evaluation of microcomputer nutritional teaching games in 1,876 children at school. Diabetes Metab 2001; 27:459-464.

11. Yien J-M, Hung C-M, Hwang G-J, Lin YC, et al. A gamebased learning approach to improving students' learning achievements in a nutrition course. TOJET 2011; 10:1-10.

12. Kreisel K. Evaluation of a computer-based nutrition education tool. Public Health Nutr 2004; 7:271-277.

13. Baños R, Cebolla A, Oliver E, et al. Efficacy and acceptability of an Internet platform to improve the learning of nutritional knowledge in children: The ETIOBE mates. Health Educ Res 2013; 28:234-248.

14. Munguba MC, Valdes MTM, Da Silva CAB. The application of an occupational therapy nutrition education programme for children who are obese. Occup Ther Int 2008; 15:56-70.

15. Johnson-Glenberg MC, Savio-Ramos C, Henry H. "Alien Health": A nutrition instruction exergame using the kinect sensor. Games Health J 2014; 3:241-251.

16. Johnson-Glenberg MC, Hekler EB. "Alien Health Game": An embodied exergame to instruct in nutrition and MyPlate. Games Health J 2013; 2:354-361.

17. Baranowski T, Blumberg F, Buday R, et al. Games for health for children-Current status and needed research. Games Health J 2016; 5:1-12.

18. Boot WR, Simons DJ, Stothart C, et al. The pervasive problem with placebos in psychology: Why active control groups are not sufficient to rule out placebo effects. Perspect Psychol Sci 2013; 8:445-454.

19. Guerrieri R, Nederkoorn C, Jansen A. The interaction between impulsivity and a varied food environment: Its influence on food intake and overweight. Int J Obes 2008; 32 : 708-714.

20. Ogden CL, Kuczmarski RJ, Flegal KM, et al. Centers for Disease Control and Prevention 2000 growth charts for the United States: Improvements to the 1977 National Center for Health Statistics version. Pediatrics 2002; 109:45-60.

21. Gruijters SL. Baseline comparisons and covariate fishing: Bad statistical habits we should have broken yesterday. Eur Health Psychol 2016; 18:205-209.

22. Cohen J. Statistical Power Analysis for the Behavioral Sciences. Hillsdale, NJ: Lawrence Earlbaum Associates; 1988.

23. Brink EJ, Postma-Smeets A, Stafleu A, et al. The Wheel of Five Factsheet. 2017. https://mobiel.voedingscentrum.nl/ Assets/Uploads/voedingscentrum/Documents/Professionals/ Pers/Factsheets/English/Fact\%20sheet\%20The\%20Wheel\% 20of\%20Five.pdf (accessed February 13, 2018).

24. Goldin-Meadow S. How gesture promotes learning throughout childhood. Child Dev Perspect 2009; 3:106111.

25. Bevelander KE, Anschütz DJ, Engels RC. Social norms in food intake among normal weight and overweight children. Appetite 2012; 58:864-872. 
26. Higgs S. Social norms and their influence on eating behaviours. Appetite 2015; 86:38-44.

27. Schoneveld E, Malmberg M, Lichtwarck-Aschoff A. A neurofeedback game (Mindlight) to prevent anxiety in children. A randomized controlled trial. Comput Hum Behav 2016; 63:321-333.

28. Contento I, Balch GI, Bronner YL, et al. The effectiveness of nutrition education and implications for nutrition education policy, programs, and research: A review of research. J Nutr Educ 1995; 27:284-418.

29. Vidgen AH, Gallegos D. Defining food literacy and its components. Appetite 2014; 76:50-59.

30. Larsen JK, Hermans RC, Sleddens EF, et al. How parental dietary behavior and food parenting practices affect children's dietary behavior. Interacting sources of influence? Appetite 2015; 89:246-257.
31. Thijssen J, Schoot FCJA, Hemker BT. Balans van het biologieonderwijs aan het einde van de basisschool 4: Extra aandachtsgebied Voeding en gezondheid: Uitkomsten van de vierde peiling in 2010. Arnhem, The Netherlands: Cito; 2011 .

Address correspondence to: Nina van den Broek, MSc Department of Developmental Psychopathology Behavioural Science Institute Radboud University Nijmegen $6500 \mathrm{HE}$ The Netherlands

E-mail: n.vandenbroek@bsi.ru.nl 